\title{
2-D Discrete Wavelet Transform for Hand Palm Texture Biometric Identification and Verification
}

\author{
Juan José Fuertes Cebrián', \\ Carlos Manuel Travieso González ${ }^{2}$ and Valery Naranjo Ornedo ${ }^{1}$ \\ ${ }^{1}$ Instituto Interuniversitario de Investigación en Bioingeniería y Tecnología Orientada al \\ Ser Humano, Universitat Politècnica de València, I3BH/Labhuman \\ 2Instituto Universitario para el Desarrollo Tecnológico y la Innovación en las \\ Comunicaciones (IDETIC), Departamento de Señales y Comunicaciones, \\ Universidad de Las Palmas de Gran Canaria
}

Spain

\section{Introduction}

In the competitive business world today, the need and demand for a biometric physical security solution has never been higher. Common biometric techniques include fingerprints, hand or palm geometry, retina, iris, or facial characteristics. Behavioural character includes signature, voice (which also has a physical component), keystroke pattern, and gait. Nowadays, most of the security systems developed into the society are based on hand image analysis (Masood et al., 2008; Pavesic et al., 2004), especially in the texture of the hand palm since they provide people with higher security in relation to authentication systems (Zhang, 2004 ; Yan \& Long, 2008) and offer a good balance of performance characteristics; besides, they are relatively easy to use. Although other biometric techniques like fingerprint or hand and finger geometry let us achieve good results, a security and highly accurate biometric system can be built through the use of palm-print texture features. The user aligns the palm of his or her hand onto a metal surface with guidance pegs that read the hand attributes of that person. Then, the device records the users' hand information and sends it to its database for identification and verification. In short, biometric identification involves determining who a person is and biometric verification determines if a person is who he/she says.

Hand palm analysis offers many advantages similar to the other technologies such as ease of use, small data collection, resistant to attempt to fool a system and unlikely technology to emulate a fake hand. In this context, wavelet analysis plays an important role in biometric systems: the wavelet transform allows to extract the main hand users' features and to differentiate people. There are however several challenges to beat.

Besides high proprietary hardware costs and size, the aging of the people hands, the lack of technology accuracy and the biometrics inability to not recognize a fake hand pose a challenge. To overcome these drawbacks, Goh et al., 2006 presented a palm-print system made up of 75 individuals. It was based on wavelet transform and Gabor filter, with a 
verification result of $96.7 \%$ and an Equal Error Rate (EER) close to $4 \%$. Liu et al., 2007, showed a research about the use of wavelet transform in palm-print. Classifying with the ISODATA algorithm got a $95 \%$ of identification accuracy with 180 palm-print of 80 people. Masood et al., 2008, developed a palm-print system using wavelet transforms. 50 people took part in the session (10 samples per person), reaching a $97.12 \%$ of accuracy with the combination of different wavelet families. Other authors have proposed different techniques of palm-print analysis: Guo et al., 2009, described a BOCV system, (Binary Orientation CoOccurrence Vector) based on the linking of six Gabor features vectors. 7752 samples from 193 people were taken. The error rate was $0.0189 \%$. Zhang et al., 2007, presented a novel 2D+3D palm-print biometric system made up to 108 individuals. The EER was $0.0022 \%$. Badrinath \& Gupta, 2009, proposed a prototype of robust biometric system for verification which uses features extracted using Speeded Up Robust Features (SURF) operator of human hand. The system was tested on IITK and PolyU database. It had FAR $=0.02 \%$, FRR $=0.01 \%$ and an accuracy of $99.98 \%$ at original size. The system addressed the robustness in the context of scale, rotation and occlusion of palm-print.

This work will address a depth study about the use of 2-D discrete wavelet transform (DWT) in order to get a simple and robust biometric identification/verification system using the texture of the hand palm. Firstly, the hand palm image processing with scale, rotation and translation invariance (ROI) is obtained. Then, we will discuss and analyze several wavelet biometric families providing its advantages and disadvantages, showing identification and verification results, and concluding with the biometrics of the future. The general block diagram of the system is shown in Figure 1. After obtaining the ROI and applying different filter levels, the recognition rate will be reached with Support Vector Machine (SVM) (Steinwart \& Christmann, 2008), a supervised classifier used to authenticate people.

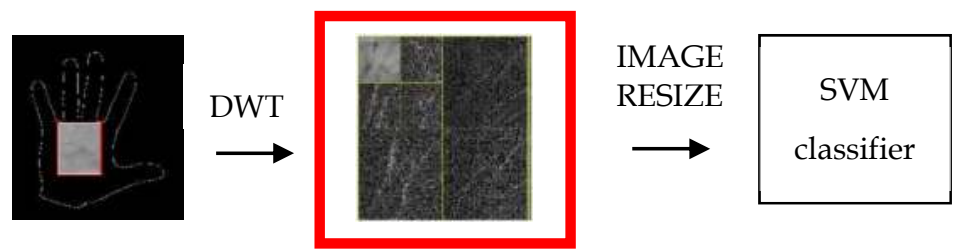

Fig. 1. Functional block diagram of the system. After extracting the palmprint, it is processed with DWT algorithm in order to get a recognition rate by means of SVM classifier.

This paper is set up as follows: section 2 reminds some basics of wavelet transform and main features of the mathematical algorithm. In section 3, the image processing, feature extraction and classification system are detailed. Section 4 shows the methodology proposed and section 5 the experiments performed. A brief comparison with methods developed by other authors is presented in section 6 and finally, the conclusion is given in section 7 together with the future work.

\section{Mathematical basis of wavelet transform}

Similarity to Fourier analysis, continuous wavelet analysis splits a signal up into several waveforms which are scaled up/down by a factor " $a$ " and displaced by a factor " $b$ ": 


$$
\psi_{a, \mathrm{~b}}=\frac{1}{\sqrt{a}} \psi\left(\frac{\mathrm{t}-\mathrm{b}}{a}\right)
$$

The result of applying wavelet analysis indicates the wavelet coefficients CWT $(a, b)$, which are dependent on the scale and the position:

$$
\operatorname{CWT}(a, b)=\mathrm{W} \cdot \mathrm{f}(a, b)=<\mathrm{f}(\mathrm{t}), \psi_{a, \mathrm{~b}}>=\int_{-\infty}^{+\infty} \mathrm{f}(\mathrm{t}) \cdot \psi^{*}\left(\frac{\mathrm{t}-\mathrm{b}}{a}\right) \mathrm{dt}
$$

If they are multiplied by the scaled and displaced wavelets, the signals can be generated. In Figure 2 the factorization of a signal in different wavelet waveforms is shown. Small " $a$ " values detect fast changes in the details, and high " $a$ " values detect slow changes.

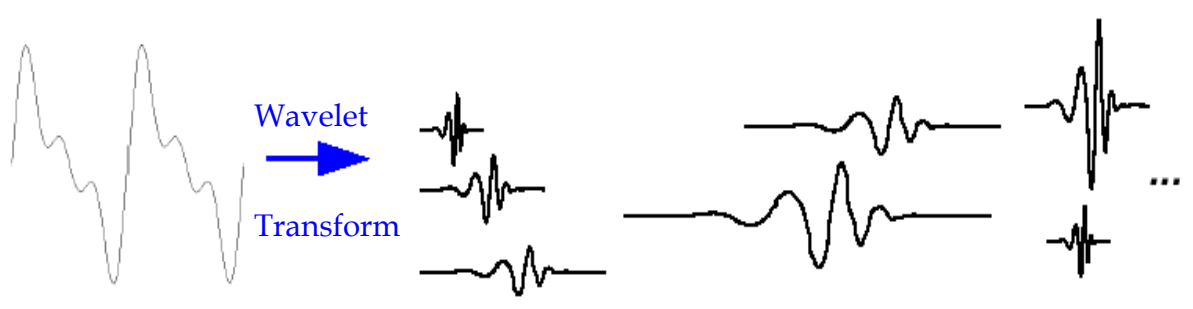

Fig. 2. An example of different wavelet waveforms.

But if we would have to calculate all the wavelet coefficients for all possible scale values, it would be costly. Therefore, in this paper the Discrete Wavelet Transform (DWT) is performed (Villegas \& Pinto, 2006), sampling the CWT in values of scale and position which are a power of two $a=2^{\mathrm{j}}, \mathrm{b}=\mathrm{k} \cdot a, \mathrm{j}, \mathrm{k} \in \mathrm{Z}$

$$
D W T(j, k)=\int_{-\infty}^{+\infty} f(t) \cdot \psi_{k}^{j}(t) d t
$$

where $\psi_{j}^{k}(t)=2^{-\frac{j}{2}} \cdot \psi\left(2^{-j} \cdot t-k\right)$.

In this work, the DWT is used to split a signal in several resolution levels (Gonzalez \& Wood, 2008). The multi-resolution split can be performed projecting the signal in two orthogonal sub-spaces called approximations and details. An efficient way to implement the DWT is using a "bank of filters". It was developed in 1989 by Mallat, whose algorithm is the diagram which is known in the signal processing community as "two channels sub-band encoder" (see Figure 3). The signal is split into two parts: low frequencies (approximations) and high frequencies (details) (Mallat, 1989). The low pass filter $H(w)$ is related to the high pass filter $G(w)$ by means of the equation:

$$
\mathrm{g}[\mathrm{L}-1-\mathrm{n}]=(-1)^{\mathrm{n}} \cdot \mathrm{h}[\mathrm{n}]
$$

where $\mathrm{L}$ is the number of levels of the bank of filters. In section 4 we will explain the use of wavelet transform, but before that, in section 3 the process to extract the hand palm image and the classification system used to get a successful recognition rate will be presented. 


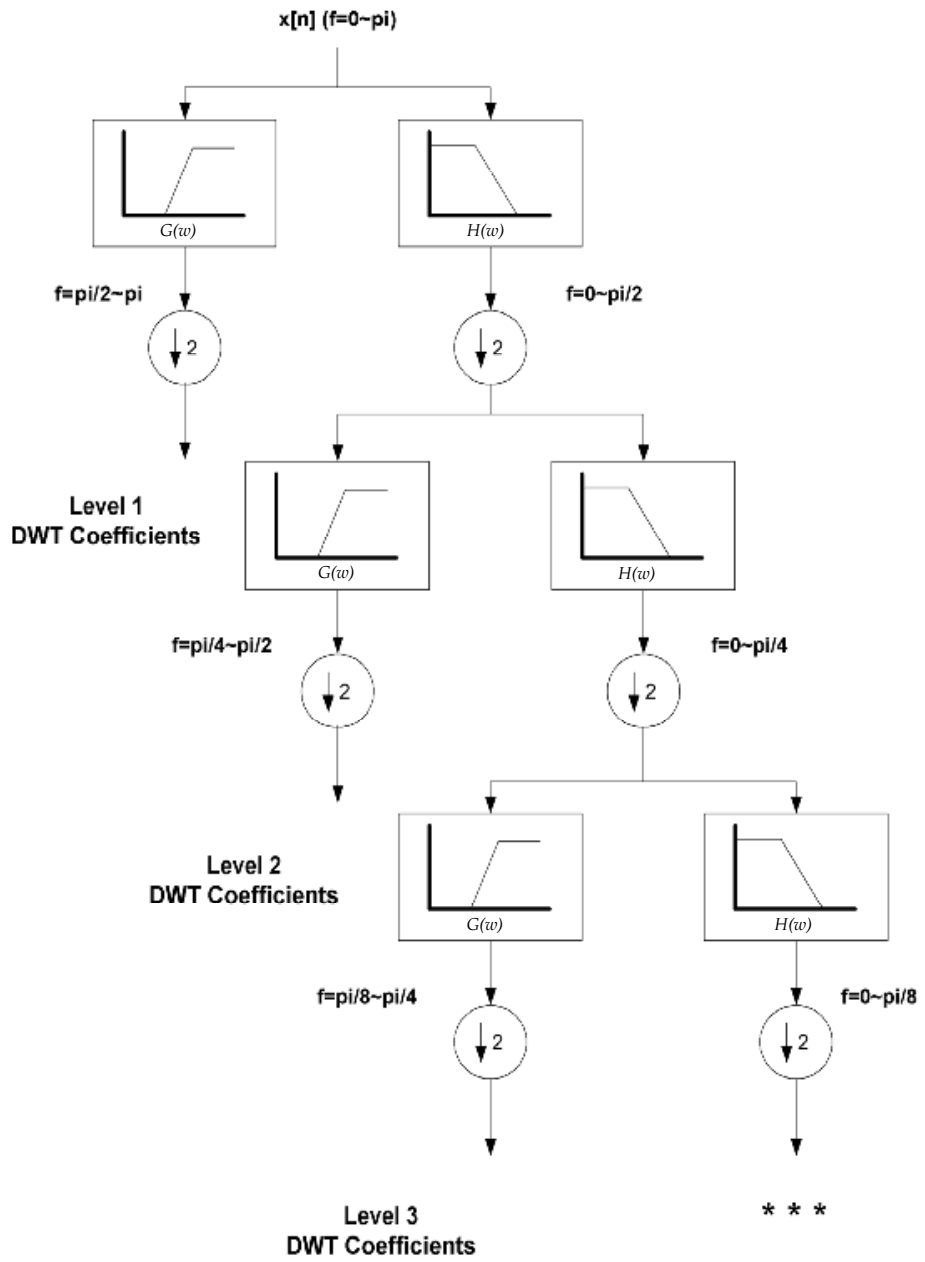

Fig. 3. Bank of filters proposed by Mallat to implement the Discrete Wavelet Transform.

\section{Image pre-processing, features extraction and classification system}

Once hand images have been acquired, each one is converted from 256 gray levels to a binary image, applying a thresholding algorithm where the threshold is obtained empirically from the training samples of the database. Then, a closing operation is performed in order to delete background noise and to select the user hand.

The goal of image pre-processing is to obtain a region of interest (ROI) with the main lines of the hand so as to extract the most important features of the user. In this way, the first step is to work out the tops and the valleys of the fingers, through the localization of the 4 fingers of the hand (through 8 initial points) with the exception of the thumb, tracing horizontal lines (see Fig. 4, Left). At this point, hand-contour is obtained subtracting the eroded from 
the dilated hand-image. Finding out the maximum of the contour between the 2 points of each finger the ends are obtained. Finding out the minimum between the 2 consecutive points of different fingers the valleys are calculated. Then, the ROI is obtained after lining up the valley of the little finger with the valley of the hearth-index finger (see Fig. 4, Right), in order to get an image with scale, rotation and translation invariance (in this work it will also be evaluated the system performance if the valleys are not aligned). The vertical size is fixed to 300 pixels and the horizontal size can vary some pixels depending on the fingers gap. Once the ROI is obtained, wavelet transform can be applied.
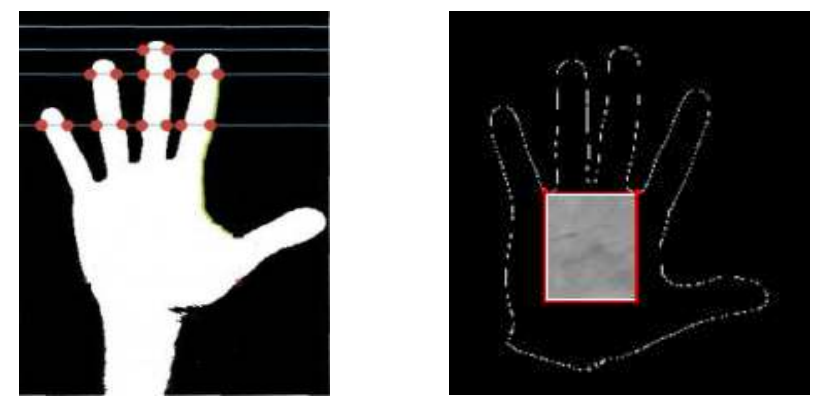

Fig. 4. Left, valleys and tops are obtained through the initial points. Right, the ROI is extracted after lining up the hand with the valleys.

A successive chain of low filters with cut-off $\mathrm{pi} / 2$ is used in order to separate the thin details from the thick details of the palm-print image. It lets to emphasize the difference between the diverse gray levels. Specifically, taking one user palmprint approximation $A_{k-1}$, we can obtain the $k$-th level decomposition applying a successive chain of filters, low pass $\left(H_{L}\right)$ or high pass $\left(H_{H}\right)$, resulting in the approximation $\left(\mathrm{A}_{\mathrm{k}}\right)$, horizontal $\left(\mathrm{H}_{\mathrm{k}}\right)$, vertical $\left(\mathrm{V}_{\mathrm{k}}\right)$ and diagonal $\left(D_{k}\right)$ detail images (Figure 5$)$. In this work, we have split the original palmprint in approximation and diagonal images, studying the response of the first one due to its high recognition rate.
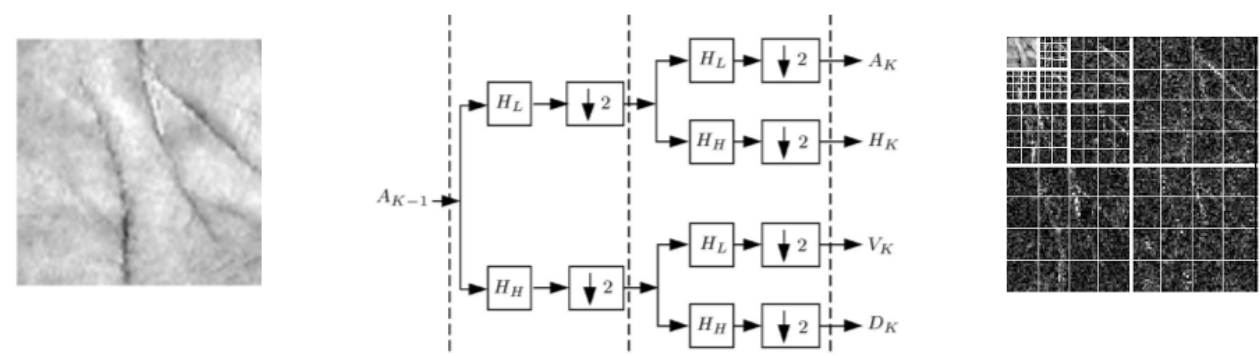

Fig. 5. Left, the original palmprint. Middle, the diagram to split de palmprint in the four images. Right, the decomposition of the palmprint in $3^{\text {rd }}$ wavelet level.

Later, the size of the resulting palm-print image can be reduced to different sizes before introducing them to the classifier in order to optimize the system performance. 


\subsection{Support Vector Machine (SVM)}

A general supervised classifier divided into two fundamental blocks is used, with the training and test block, just as it is shown in the Figure 6. In that figure, the classification process is shown after obtaining and extracting the data. With the training parameters, a score feature which is used in the test step is modelled.

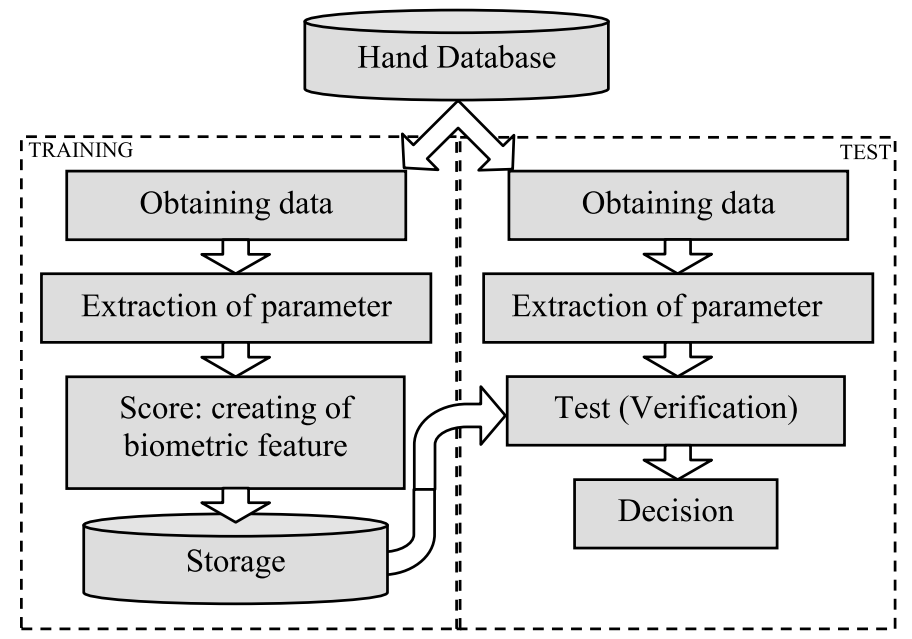

Fig. 6. Block diagram of the supervised identification/verification system. Left, training block; right, test block.

To evaluate the hand features, Support Vector Machines (SVM, Steinwart \& Christmann, 2008) are used when they work as identifier or verifier, where only known users appear in the test data (closed mode). At that moment, linear (Figure 7) or RBF kernel (Figure 8) can be used to verify/identify users, if the data are or not linearly separable.

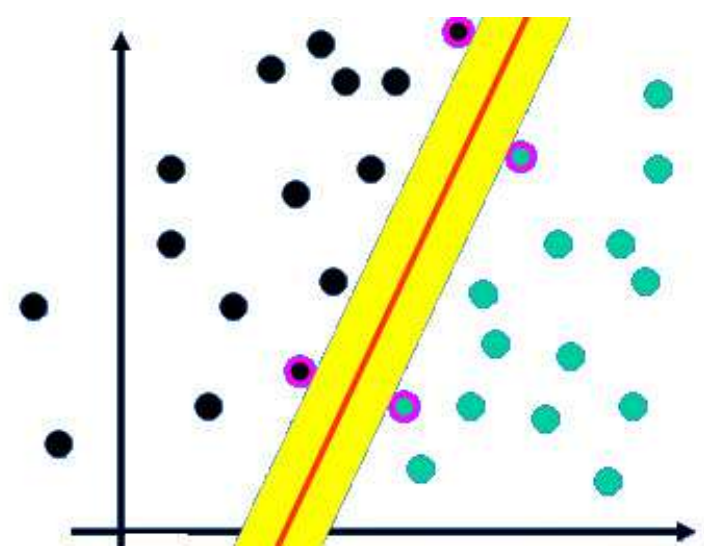

Fig. 7. Maximization of the hyperplane with linear kernel. 


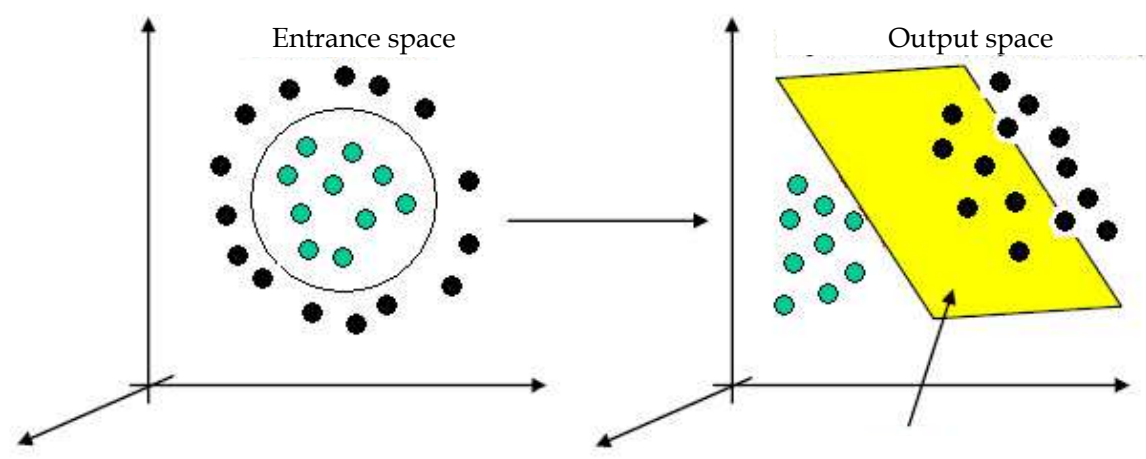

Hyperplane

Fig. 8. Data transformation to higher space with RBF kernel.

Analyzing Figures 7 and 8, it would be possible to say that SVM builds a hyperplane of separation in the entrance space in two possible modes: in the first, the input space is converted into higher dimension characteristics space, by means of a (nucleus or kernel) (non)- linear transformation; in the second, the optimum hyperplane of separation (MMH, Maximal Margin Hyperplane) is built. This hyperplane maximizes the distance of the vectors which belong to different classes. Thus, if $S$ is a set of $N$ vectors $\boldsymbol{x}_{\mathbf{i}} € \mathrm{R}^{\mathrm{n}}$ where $\mathrm{i}=1 \ldots \mathrm{N}$, each vector $\boldsymbol{x}_{\mathbf{i}}$ belongs to one of the two identifying classes as $y_{\mathrm{i}} \in\{-1,1\}$. If the two classes are linearly separable, a unique optimum hyperplane exists and it is defined by:

$$
\boldsymbol{w} \cdot \boldsymbol{x}+\mathrm{b}=0
$$

where $\boldsymbol{w}$ denotes the normal vector to the hyperplane and $b /\|\boldsymbol{w}\|$ is the distance between the origin and the hyperplane. It provides a greater margin of separation among the classes, and it divides $\mathbf{S}$ leaving all the vectors of the same class in the same side of the hyperplane. This hyperplane can be obtained resolving the problem,

$$
\begin{array}{cc}
\multicolumn{1}{c}{\text { minimize }} & g(\boldsymbol{w})=\frac{1}{2}\|\boldsymbol{w}\|^{2} \\
\text { subject to } & y_{\mathrm{i}}\left(\boldsymbol{w} \cdot \boldsymbol{x}_{\boldsymbol{i}}+\mathrm{b}\right) \geq 1, \quad i=1, \ldots, N
\end{array}
$$

Therefore, it treats a problem of square programming whose solution is obtained applying the theorem of Karush-Kuhn-Tueker, which can be written as:

$$
\boldsymbol{w}=\sum_{i=1}^{N} \alpha_{i} y_{i} \boldsymbol{x}_{\boldsymbol{i}}
$$

The associated vectors to the multiplicities of Lagrange $\left(\alpha_{i}\right)$ which are not null are called support vectors, and they are the unique vectors of $S$ that are necessary to determine the optimum hyperplane. To extend the concept of SVM to non linear classifiers, it is carried out a transformation of the input space to other space of higher dimension, in which the data are linearly separable. Thus, each input vector is transformed into other vector $\mathbf{z}=\Phi(\mathbf{z})$ of greater dimension in the space of characteristics. Under certain conditions, given by the theorem of Mercer (Mercer et al., 1999), the scale product in the space of characteristics can be expressed by a certain nucleus $K(\boldsymbol{x}, \boldsymbol{y})$. In this case, the problem is reduced to resolve; 


$$
\begin{array}{cc}
\text { minimize } & g(\boldsymbol{w})=\frac{1}{2}\|\boldsymbol{w}\|^{2}+C \sum_{i=1}^{N} \xi_{i} \\
\text { subject to } & y_{i}\left(\boldsymbol{w} \cdot \boldsymbol{\Phi}\left(\boldsymbol{x}_{\boldsymbol{i}}\right)+\mathrm{b}\right) \geq 1-\xi_{i}, \quad i=1, \ldots, N
\end{array}
$$

where $\xi_{i}$ are lack variables which measure the degree of misclassification of the datum $\boldsymbol{x}_{\boldsymbol{i}}$, and the vector $\boldsymbol{w}$ can be obtained again, as combination of the transformed support vectors. Support Vector Machines (SVM) are based on a bi-class system, where only two classes are considered. In this work, we have worked on identification and verification system, and for this reason, a one-versus-all and one-versus-one strategy are built respectively. To express the similarity between biometric patterns, in our case palm-print modality, the Recognition Rate (RR) in identifier and the Equal Error Rate (EER) in verifier mode are used. The higher the RR, the better the identifier performance.

In verifier mode, the EER of a system can be used to give a threshold independent performance measure (or TEER - Threshold of Equal Error Rate). The lower the EER, the better the verifier performance, since the total error rate which is the sum of the FAR (False Acceptance Rate) and the FRR (False Rejection Rate) at the point of the EER decreases. In theory, this work is fine if the EER of the system is calculated using an infinite and representative test set, but it is not possible under real world conditions. Therefore, to get comparable results it is necessary that the EERs, which are compared, are calculated on the same test data using the same test protocol. In our experiments, ERR and its TEER have been calculated.

\section{Experimental methodology}

The 1440 hand-images belonging to the 144 users of the database are acquired thanks to a 150 dots per inch general scanner putting the users' hand on its surface. Then, they are stored with 256 gray levels, 8 bits per pixel. The size of these images is set to $1403 \times 1021$ pixels after scaling them by a factor of $20 \%$ to facilitate later computation (See Table 1 ).

\begin{tabular}{lc}
\hline Properties of the hand images contained in the database \\
\hline Size & $80 \%$ original size \\
Resolution & $150 \mathrm{dpi}$ \\
Colour & 256 grey levels \\
File size & 1405 Kbytes \\
Data matrix dimension & $1403 \times 1021$ \\
\hline
\end{tabular}

Table 1. Image Specifications.

Four experiments are performed with the database hand images.

1. In the first experiment, the recognition rate (RR) for 50 users is shown, after applying from 1 to 4 filter levels for each wavelet family above straight hand palm image (ROI obtained without aligning the valleys).

2. In the second experiment, the wavelet algorithm is evaluated above the sloping ROI (after aligning the valleys of the hand), under the same conditions in order to compare both methods. 
3. In the third experiment, the recognition rate for straight and sloping ROI above the 144 users of the database to observe the dependence they have on the system is presented.

4. Finally, in the last experiment the best algorithm performance when the classifier works as verifier is shown. Besides, the False Acceptance Rate, the False Rejection Rate and the Equal Error Rate are detailed.

In order to optimize the results of these experiments, the research covers from the data analysis to classifier selection, studying several hand image sizes, DWT family, filter levels, RBF gamma values and the system performance when it works as identifier and verifier. In Figure 9 the different parameters studied in order are shown.

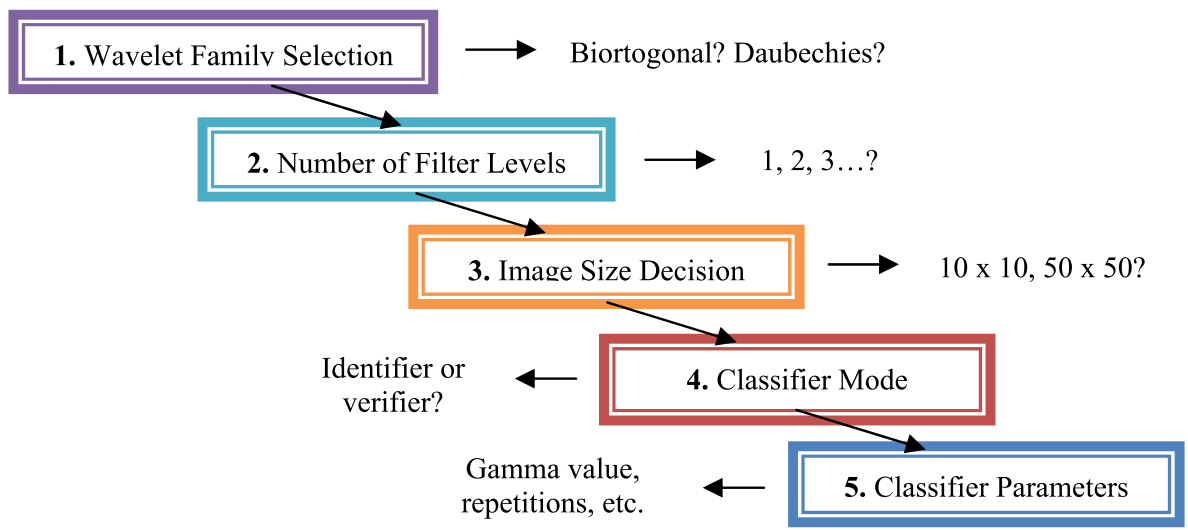

Fig. 9. The blocks and parameters studied to evaluate system accuracy.

The first step consists of the kind of filter and the number of levels to apply over the images. In this paper three wavelet families have been compared: 'haar' or 'db1', 'daubechies5' and 'biortogonal5.5' (see Figure 10).
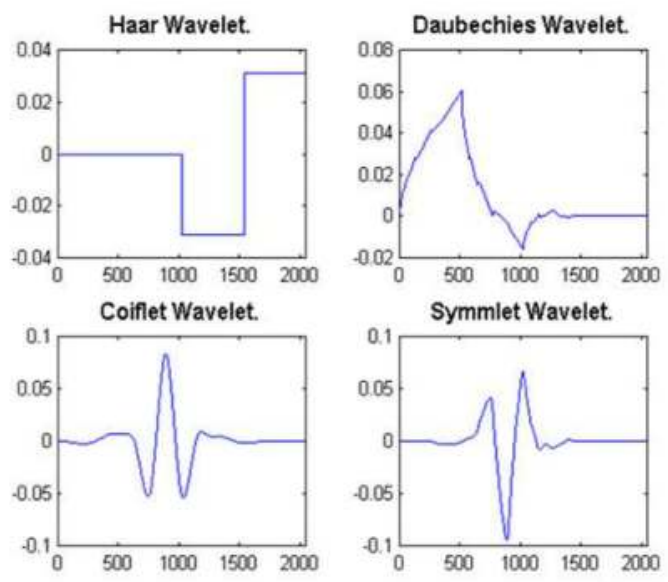

Fig. 10. Several Wavelet families: haar, daubechies, coiflet and symmlet. 
The number of filter levels is varied from 1 to 4 , but when 1 or 4 filters are applied the recognition rate decreases considerably. To facilitate the image filtering, it has been used a successive chain of low filters with cut-off pi/2 in order to separate the thin details from the thick details of the palmprint image, as it has been explained in section 2.

After the hand image is filtered, it can be rescaled to a particular size. However, best results are obtained after applying three consecutive low filters to the ROI, without reducing the hand-palm size (original size after the filters) as it will show in results section.

At this point, the classifier works as identifier or verifier. When it works as identifier, the system recognizes the identity of the person whose distance to the hyperplane is largest. If the system works as verifier, it accepts the identity of the person when the distance to the hyperplane is greater than the optimum threshold calculated in training step (Adán et al., 2008). Finally, the gamma value of the classifier is changed to get the best recognition rate.

\section{Identification/verification results for biortogonal, daubechies and haar wavelet families}

As it was introduced in the previous section, we have collected 1440 samples from 144 users, 10 samples per user (see Table I to know image specifications). Four images of each user are chosen randomly as training samples and the remaining six images are used to test the system. At this time, the extracted and filtered palmprint is introduced to the classifier in order to get the recognition rate. The results are shown in average (\%) and typical deviation (std).

Each test has been done 10 times using linear and RBF (radial basis functions) SVM kernel, finding the final result through a cross-validation strategy. The supervised classification has been carried out with the library SVM_light (Cortes \& Vapnik, 1995). In all experiments, we have evaluated the system in closed mode (only known users appear in the test data), where 50 or 144 users have been used as test and training data.

In Figures 11, 12 and 13, the recognition rates (along x-axis) of wavelet haar, daubechies5 and biortogonal5 families (experiment number 1) for different size palm image reductions (along y-axis) are shown. The number of filter levels is varied from 1 to 4 when the system works as identifier for 50 users (RBF classifier).
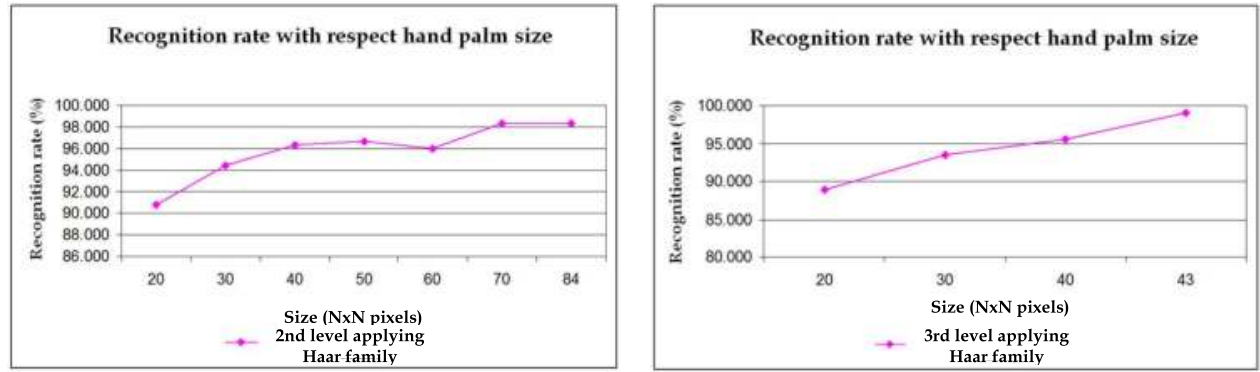

Fig. 11. Recognition rate with Haar Wavelet filter. 

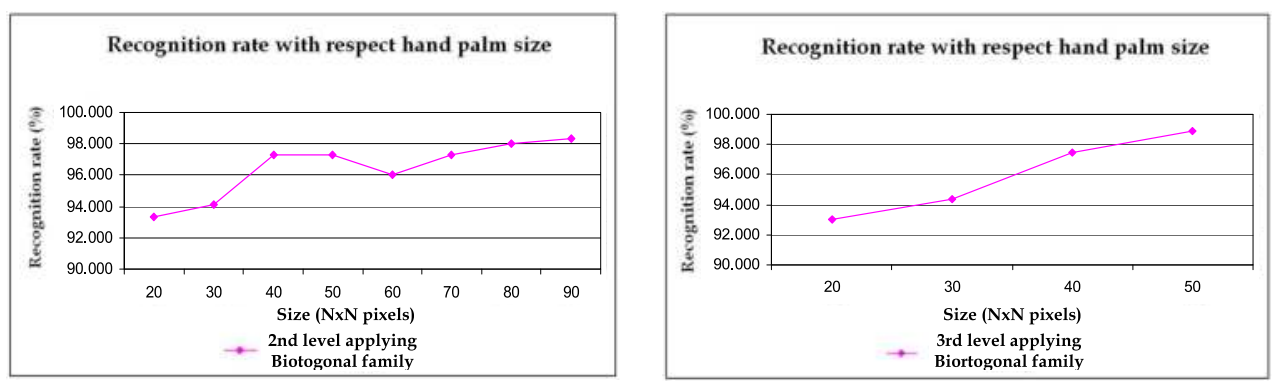

Fig. 12. Recognition rate with Biortogonal Wavelet filter.
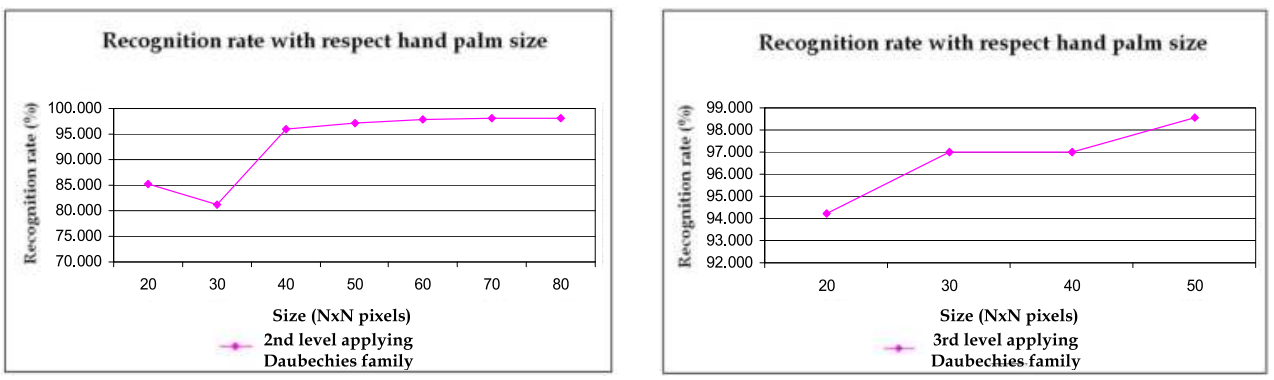

Fig. 13. Recognition rate with Daubechies Wavelet filter.

When the number of levels of the filter was 1 or 4 , the recognition rate decreased drastically up to $85 \%$ approximately, with both linear and RBF kernels. In all cases, the best recognition rate is obtained when a 3rd filter level is applied without reducing the resulting images size. This happens because in the second and specifically in the third level, the information available to maximize the interclass relationship is highest.

Analyzing the figures, the higher the palmprint size, the higher the recognition rate. This happens because important discriminative features are ruled out when the image is reduced to a small size. The size is not increased either, since it supposes a higher computational cost and the recognition rate keeps constant. Moreover, when the wavelet family is the Biortogonal5.5, the recognition rate is higher than with the other signal families. When the applied filter is daubechies5 or haar, the recognition rate progress is similar to biortogonal5.5 filter.

Before testing the wavelet filter for the full database, it was applied the algorithm over the SLOPING ROI for 50 users (experiment number 2) in order to evaluate the influence of preprocessing steps (aligning the valleys).

As Table 2 shows, the results obtained for the three wavelet families are similar either linear or RBF kernel. For this reason, the three wavelet families must be evaluated for 144 people. In Table 3 the highest recognition rate with linear and RBF kernels for 144 users in identifier mode is shown (experiment number 3). In this case, the results demonstrate the difference when the ROI is aligned with its valleys (SLOPING ROI) or not (STRAIGHT ROI). 


\begin{tabular}{|c|c|c|}
\hline \multicolumn{3}{|c|}{ IDENTIFIER - 50 USERS } \\
\hline Features & Linear recognition rate & RBF recognition rate \\
\hline Wavelet Haar 3 filters & $99.33 \% \pm 0.00$ & $99.17 \% \pm 0.06$ \\
\hline $\begin{array}{c}\text { Wavelet db5 } \\
\text { 3 filters }\end{array}$ & $99.66 \% \pm 0.22$ & $99.50 \% \pm 0.06$ \\
\hline $\begin{array}{c}\text { Wavelet bior5.5 } \\
\text { 3 filters }\end{array}$ & $99.83 \% \pm 0.06$ & $99.67 \% \pm 0.22$ \\
\hline
\end{tabular}

Table 2. Wavelet identification results for 50 users using the sloping ROI.

\begin{tabular}{|c|c|c|c|c|}
\hline \multicolumn{3}{|c|}{ STRAIGHT ROI - IDENTIFIER } & \multicolumn{2}{c|}{ SLOPING ROI - IDENTIFIER } \\
\hline Features & $\begin{array}{c}\text { Linear } \\
\text { recognition }\end{array}$ & RBF recognition & Linear recognition & RBF recognition \\
\hline $\begin{array}{c}\text { haar } \\
\text { 3 filter levels }\end{array}$ & $99.11 \% \pm 0.59$ & $99.00 \% \pm 0.77$ & $99.54 \% \pm 0.01$ & $99.73 \% \pm 0.03$ \\
\hline $\begin{array}{c}\text { db5 } \\
\text { 3 filter levels }\end{array}$ & $98.44 \% \pm 1.59$ & $98.55 \% \pm 1.15$ & $99.54 \% \pm 0.01$ & $99.31 \% \pm 0.12$ \\
\hline $\begin{array}{c}\text { bior5.5 } \\
\text { f filter levels }\end{array}$ & $98.77 \% \pm 0.26$ & $98.88 \% \pm 0.26$ & $99.76 \% \pm 0.01$ & $99.65 \% \pm 0.02$ \\
\hline
\end{tabular}

Table 3. Wavelet identification results for 144 users using the straight and sloping ROI.

According to tables 2 and 3, the recognition rate is similar regardless of database users' number, unlike other texture algorithms such as derivative method or Gabor filter (Fuertes et al., 2010). The best recognition rate for 144 users $(99.76 \% \pm 0.01)$ is reached with linear kernel for biortogonal5.5 wavelet. This result is similar to $99.83 \%$ reached for 50 users. The third level of the low pass filter provides the suitable value of the texture to maximize the interclass relationship in order to get the large discriminative information. This is possible because it makes better use of the frequency-space resolution, and consequently the classifier is able to differentiate the users properly.

In Table 4, the EER for the best method (biortogonal wavelet family) is shown. The experiment is performed after applying 3 filters when the system works as verifier (experiment number 4). For a threshold of -0.5400 , the EER is $0.60 \%$. This result agrees with others proposed in the literature, although a higher accuracy system must be built with the combination of other techniques.

\begin{tabular}{|c|c|c|c|}
\hline \multicolumn{4}{|c|}{ VERIFIER - 144 USERS } \\
\hline Features & Threshold & Error average (percentage) & EER \\
\hline \multirow{9}{*}{$\begin{array}{l}\text { Wavelet } 3 \text { filter levels } \\
\text { (bior5.5) }\end{array}$} & -0.6120 & $0.70 \%$ & \multirow{9}{*}{$0.60 \%$} \\
\hline & -0.5760 & $0.65 \%$ & \\
\hline & -0.5400 & $0.60 \%$ & \\
\hline & -0.5040 & $0.58 \%$ & \\
\hline & -0.4680 & $0.59 \%$ & \\
\hline & -0.5760 & $0.65 \%$ & \\
\hline & -0.5400 & $0.66 \%$ & \\
\hline & -0.5040 & $0.64 \%$ & \\
\hline & -0.4680 & $0.65 \%$ & \\
\hline
\end{tabular}

Table 4. Wavelet verification results for 144 users using the sloping ROI. 
In Figure 14 it is also shown how the FAR (False Acceptance Rate) and the FRR (False Rejecting Rate) change depending on the threshold.

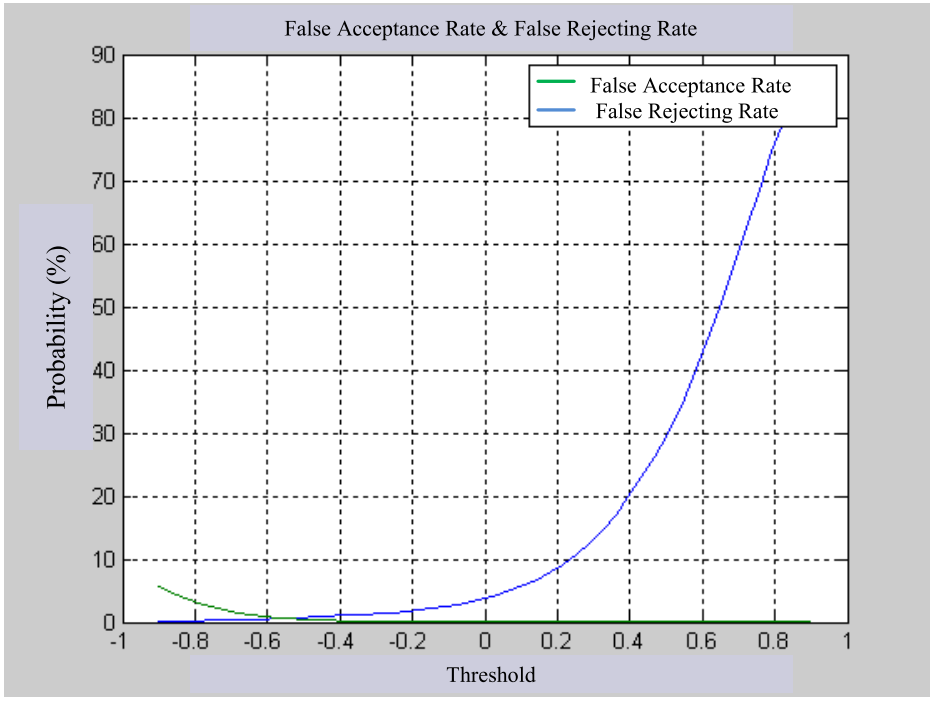

Fig. 14. FAR, FRR and EER for 144 database users.

As Figure 14 shows, the FAR and FRR curves decrease depending on threshold value. The point where both curves intersect other define the best accuracy of the verifier system. In next section we will discuss about these results and the system performance. It will be also introduced the future work and possible improvements in our work.

\section{Comparison with other methods and discussion}

In this section a brief comparison with wavelet experiments performed by other authors is detailed. Table 5 shows the recognition rate when the system works as identifier for several wavelet analysis which have been introduced in section 1 .

\begin{tabular}{|c|c|c|c|c|}
\hline Authors & Features & Users & $\begin{array}{c}\text { Samples by } \\
\text { user }\end{array}$ & $\begin{array}{c}\text { Recognition } \\
\text { rate }\end{array}$ \\
\hline This work & $\begin{array}{c}\text { Wavelet bior5.5 } \\
\text { 3 filter levels }\end{array}$ & 144 & 10 & $99.76 \%$ \\
\hline $\begin{array}{c}\text { Xian-Qian et } \\
\text { al., 2002 }\end{array}$ & Wavelet 3 filter levels Haar & 200 & 5 & $99 \%$ \\
\hline $\begin{array}{c}\text { Masood et } \\
\text { al., 2008 }\end{array}$ & $\begin{array}{c}\text { Wavelet, biortogonal, symlet } \\
\text { y meyer }\end{array}$ & 50 & 10 & $97.12 \%$ \\
\hline $\begin{array}{c}\text { Liu et al., } \\
2007\end{array}$ & Wavelet (ISODATA) & 80 & $\begin{array}{c}180 \\
\text { (altogether) }\end{array}$ & $95 \%$ \\
\hline
\end{tabular}

Table 5. Identification results published by other authors about wavelet transform. 
A comparison with other texture-geometry techniques performed in the past by the authors of this work is also shown in Table 6 (Fuertes et al., 2010). In this case, a recognition rate of $99.73 \%$ was obtained with the Gabor algorithm and a $99.46 \%$ with the derivative method. In addition, a $99.90 \%$ was reached with the study of the hand geometry, especially with the finger widths.

\begin{tabular}{|c|c|c|}
\hline $\begin{array}{c}\text { Methods (Fuertes et } \\
\text { al. 2010) }\end{array}$ & Linear recognition & RBF recognition \\
\hline 2D Gabor & $99.73 \% \pm 0.03$ & $99.73 \% \pm 0.03$ \\
\hline Derivative Method & $99.46 \% \pm 0.09$ & $99.46 \% \pm 0.13$ \\
\hline $\begin{array}{c}\text { 4 fingers(20 widths } \\
\text { each finger) }\end{array}$ & $99.38 \% \pm 0.02$ & $99.90 \% \pm 0.01$ \\
\hline
\end{tabular}

Table 6. Identification results published by the authors of this work about other hand image techniques.

The results obtained in this work agree with others proposed in the literature. The best texture method, the biortogonal (bior5.5) wavelet, reaches a value similar to the obtained with the haar or db5 wavelet families. In Table 6, the results of the best methods of geometry and other texture methods performed in the past also agree with wavelet analysis. A combination of all these methods would improve the system recognition.

The conclusions arising from the experiments and its results will be explained in section 7 , considering the future work and several improvements.

\section{Conclusions and future work}

In this paper, the performance of 2-D wavelet transform for hand palm biometric system has been presented with the intention of contributing to biometric field.

The best result of the identification system is obtained when the biortogonal5.5 wavelet is performed, with a recognition rate of $99.76 \%$ for the sloping ROI. These algorithms were applied to 50 and 144 users, and the recognition rate was similar independently of the number of users. It lets the system be useful for different number of people. In addition, the hand palm texture extraction algorithm is intuitive, simple and quick, with a computational load similar to geometrical parameter extraction.

It is crucial to emphasize the importance when the ROI is obtained. It has to be extracted after aligning the valleys of the hand: in this way, the area to study covers the main hand lines, minimizing the zones where the noise is higher. This issue has been proved with the experiments performed.

If the system works as verifier, the EER is $0.60 \%$. There are other algorithms with best performance, but the possibility to combine it with other techniques makes wavelet algorithm suitable for verify people. We propose the combination of the wavelet method, proposed in this work, with other palm-print algorithms as derivative or Gabor analysis or with other hand recognition algorithms applying several fusion levels (Ferrer et al., 2007, Morales et al., 2008).

The proposed biometric feature is well adapted to the SVM classifier, which identify the feature degree of simplification necessary for the best performance. One drawback we have 
to mention is the necessity of operating with clean hands. Painted or dirty hands would cause an identification mistake. In this case a combined geometric/palm biometric feature would be more advisable.

Specifically for this research line, it will be interesting a deep study about the stability of the wavelet algorithm for many users. It is not the same to test a system with 100 or 1000 users in order to get a system regardless of number of users. If the number of users is long and unknown, wavelet analysis can be an excellent technique to recognize people.

The future of hand scanning appears to be static. Public acceptance of biometrics is growing slowly, until issues about privacy and security bring up to an acceptable level by majority of people. From a cost standpoint, hand image analysis is more expensive than fingerprint technology and just as effective. If it is used in conjunction with other techniques, passwords, and smart card and tokens then, it could prove to be a viable method. "Recent surveys suggest people are feeling more comfortable using biometric security, which could result in a $\$ 3$ billion spending increase in biometrics over the next years" (Security world news, 2010).

One way to overcome this scepticism is to use new multimodal techniques (using more than one biometric system simultaneously to confirm identification), as vein impression or 3D facial recognition. Besides, a new method as tongue scanning identification is being tested at the Hong Kong Polytechnic University's Biometrics Research Centre. The tongue shapes of different people are different, and thus the tongue can be used to tell different subjects.

Future experiments about the use of free-contact systems and the combination of palmprint analysis with emergent techniques will be performed in order to contribute to scientific spreading.

\section{Acknowledgements}

This work has been partially supported by "Catedra Telefónica - ULPGC 2009/10" (Spanish Company), and partially supported by Spanish Government under funds from MCINN TEC2009-14123-C04-01. Authors want to thanks to Processing Digital Signal Division from Institute for Technological Development and Innovation in Communications (PDSD-IDETIC) for the work which all the Division has done on the building of the Palm-print database.

\section{References}

Adán, M.; Adán, A.; Vázquez, A.S. \& Torres, R. (2008). Biometric verification/identification based on hands natural layout. Image and Vision Computing, Vol. 26, No. 4, pp. 451465, April 2008.

Badrinath, G. \& Gupta, P. (2009). Robust Biometric System Using Palmprint for Personal Verification. Advances in Biometrics, Lecture Notes in Computer Science, Vol. 5558, pp. 554-565, 2009.

Cortes, C. \& Vapnik, V. (1995). Support Vector Networks. Machine Learning, Vol. 20, pp. 273-297, 1995.

Ferrer, M.A.; Morales, A.; Travieso, C.M. \& Alonso, J.B. (2007). Low Cost Multimodal Biometric identification System Based on Hand Geometry, Palm and Finger Print Texture. 41st Annual IEEE International Carnahan Conference on Security Technology, pp. 52-58, 2007. 
Fuertes, J.J.; Travieso, C.M.; Ferrer, M.A. \& Alonso, J.B. (2010). Intra-modal biometric system using hand-geometry and palmprint texture. Security Technology (ICCST), 2010 IEEE International Carnahan Conference on, pp. 318-322, October 2010.

Goh, M.K.; Connie, T.; Teoh, A.B.J. \& Ngo, D.C.L. (2006). A Fast Palm Print Verification System. Proceedings of the International Conference on Computer Graphics, Imaging and Visualization, pp. 168-172, July 2006.

Gonzalez, R.C. \& Wood, R.E. (2008). Digital Image Processing, 3rd ed., Prentice Hall, ISBN 9780-131687288 Upper Saddle River, NJ, 2008.

Guo, Z.; Zhang, D., Zhang, L. \& Zuo, W. (2009). Palmprint verification using binary orientation co-occurrence vector. Pattern Recognition Letters, Vol. 30, No. 13, pp. 1219-1227, May 2009.

Liu, F.; Lin, C.X.; Cui, P.Y. \& Dong, T. (2007). Palmprint recognition based on ISODATA clustering algorithm. Proceedings of the 2007 International Conference on Wavelet Analysis and Pattern Recognition, Vol.3, pp. 1129-1133, Beijing, China, 2-4 Nov. 2007.

Mallat, S.G. (1989). A Theory for Multiresolution Signal Decomposition: The Wavelet Representation, IEEE Transactions on Pattern Analysis and Machine Intelligence, Vol.11, No. 7, pp. 674-693, ISSN 0162-8828, 1989.

Masood, H. ; Mumtaz, M.; Butt, M.A.A. ; Mansoor, A.B. \& Khan, S.A. (2008). Wavelet Based Palmprint Authentication System, Proceedings of IEEE International Symposium of Biometric and Security Technologies, 1-7, Islamabad, Pakistan, April 2008.

Mercer Schöfkopf, B.; Burges, C. \& Smola, A. (1999). Pairwise Classification and Support Vector Machines, The MIT Press, Cambridge, Massachusetts, pp. 255-268, 1999.

Morales, A.;Ferrer, M.; Díaz, F.; Alonso, J. \& Travieso, C. (2008). Contact-free hand biometric system for real environments. Proceedings of the 16th European Signal Processing Conference (EUSIPCO), Laussane, Switzerland, September 2008.

Pavesic, N. ; Ribaric, S. \& Ribaric, D. (2004). Personal Authentication Using Hand-Geometry and Palmprint Features, Proceedings of the Workshop on Biometrics at ICPR04, Cambridge, UK, 2004.

Security World News. (2010). http://www.securityworldnews.com/2010/02/07/the-future-ofbiometrics/ Last visit: 01-09-2011.

Steinwart, I. \& Christmann, A. (2008). Support Vector Machines, Information Science and Statistics, Springer, ISBN 978-0-387-77241-7, New York, 2008.

Villegas, O.O.V. \& Pinto R.E. (2006). Procesamiento Digital de Imágenes en el Dominio Wavelet, IEEE Looking Forward, Vol. 13, pp. 13-16, IEEE Computer Society, 2006.

Xian Qian W.; Zhang, D. \& Wang, K. (2002). Wavelet based palmprint recognition. Proceedings of the First International Conference on Machine Learning and Cybernetics, Vol. 3, pp. 1253-1257, Beijing, 4-5 November 2002.

Yan, H. \& Long, D. (2008). A novel bimodal identification approach based on hand-print. Proceedings of the 2008 Congress on Image and Signal Processing, Vol.4, 506-510, 2008.

Zhang, D.D. (2004). Palmprint Authentication, International Series on Biometrics, Vol.3, 256 p., 2004

Zhang, L.; Guo, Z.; Wang, Z. \& Zhang, D. (2007). Palmprint Verification using Complex Wavelet Transform. IEEE International Conference on Image Processing, Vol.2, pp. 417420, 2007.

Zhang, D.; Kanhangad, V.; Luo, N. \& Kumar, A. (2010). Robust palm print verification using 2D and 3D features. Journal on Pattern Recognition, Vol. 43, No. 1, pp. 358-368, January 2010. 


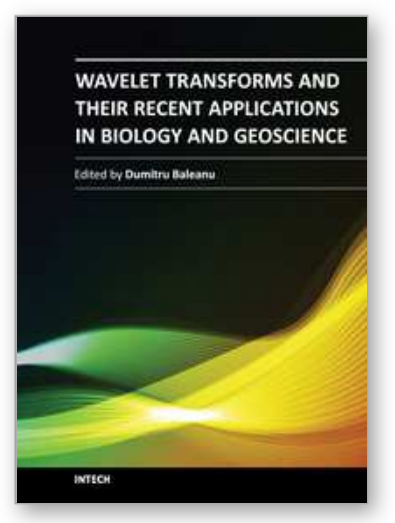

\section{Wavelet Transforms and Their Recent Applications in Biology and Geoscience}

Edited by Dr. Dumitru Baleanu

ISBN 978-953-51-0212-0

Hard cover, 298 pages

Publisher InTech

Published online 02, March, 2012

Published in print edition March, 2012

This book reports on recent applications in biology and geoscience. Among them we mention the application of wavelet transforms in the treatment of EEG signals, the dimensionality reduction of the gait recognition framework, the biometric identification and verification. The book also contains applications of the wavelet transforms in the analysis of data collected from sport and breast cancer. The denoting procedure is analyzed within wavelet transform and applied on data coming from real world applications. The book ends with two important applications of the wavelet transforms in geoscience.

\section{How to reference}

In order to correctly reference this scholarly work, feel free to copy and paste the following:

Juan José Fuertes Cebrián, Carlos Manuel Travieso González and Valery Naranjo Ornedo (2012). 2-D Discrete Wavelet Transform for Hand Palm Texture Biometric Identification and Verification, Wavelet Transforms and Their Recent Applications in Biology and Geoscience, Dr. Dumitru Baleanu (Ed.), ISBN: 978953-51-0212-0, InTech, Available from: http://www.intechopen.com/books/wavelet-transforms-and-theirrecent-applications-in-biology-and-geoscience/2-d-discrete-wavelet-transform-for-hand-palm-texturebiometric-identification-and-verification

\section{INTECH}

open science | open minds

\section{InTech Europe}

University Campus STeP Ri

Slavka Krautzeka 83/A

51000 Rijeka, Croatia

Phone: +385 (51) 770447

Fax: +385 (51) 686166

www.intechopen.com

\section{InTech China}

Unit 405, Office Block, Hotel Equatorial Shanghai

No.65, Yan An Road (West), Shanghai, 200040, China 中国上海市延安西路65号上海国际贵都大饭店办公楼 405 单元

Phone: +86-21-62489820

Fax: +86-21-62489821 
(C) 2012 The Author(s). Licensee IntechOpen. This is an open access article distributed under the terms of the Creative Commons Attribution 3.0 License, which permits unrestricted use, distribution, and reproduction in any medium, provided the original work is properly cited. 